\title{
SiECA: Silicon Photomultiplier Prototype for Flight with EUSO-SPB
}

\author{
William Painter \\ KIT-IEKP, Karlsruhe Institute of Technology, Germany \\ E-mail: william.painter@kit.edu
}

\section{Mario Bertaina}

Department of Physics, University of Torino \& INFN Torino, Italy

E-mail: bertaina@to.infn.it

\begin{abstract}
Alberto Bortone
Department of Physics, University of Torino, Italy

E-mail: alberto.bortonededu.unito.it
\end{abstract}

\section{Andreas Haungs*}

KIT-IKP, Karlsruhe Institute of Technology, Germany

E-mail: andreas.haungs@kit.edu

\section{Alexander Menshikov}

KIT-IPE, Karlsruhe Institute of Technology, Germany

E-mail: alexander.menshikov@kit.edu

\section{Max Renschler}

KIT-IKP, Karlsruhe Institute of Technology, Germany

E-mail: max.renschlerakit.edu

\section{for the JEM-EUSO Collaboration}

Silicon Photomultipliers (SiPM or MPPC) offer an alternative low light sensor to Multi-Anode Photomultiplier Tubes with the benefits of low operating voltage, compact packaging and reduced susceptibility to damage. The Silicon photomultiplier Elementary Cell Add-on camera (SiECA) comprises front end opto-electric sensors, SiPM specific ASIC signal processing chipset, data handling FPGA, support electronics and firmware integrated in a robust unit for integration on existing detector systems. This paper presents the technical aspects of using SiPM or MPPC for astroparticle physics, particularly with respect to this implementation. A first flight of this camera was made with the EUSO-SPB mission starting April 2017.

35th International Cosmic Ray Conference - ICRC2017-

10-20 July, 2017

Bexco, Busan, Korea

\footnotetext{
*Speaker.
} 


\section{Introduction}

Silicon photomultipliers (SiPM) are the solid-state, small form, lower operating voltage, replacement for high voltage photomultipliers. Use of high density, photomultiplier arrays, Multi-Anode Photo-Multiplier Tubes (MAPMTs) in the indirect detection of high and ultra high energy cosmic ray's (UHECR) fluorescence and Cherenkov emission is well established [1]. Additionally, development of terrestrial detectors utilizing SiPM is ongoing within multiple collaborative programs including the Auger Muons and Infill for the Ground Array (AMIGA) [2], Cherenkov Telescope Array (CTA) [3], FAMOUS [4], IceTop-Gen2 Scintillator Upgrade [5], etc. Thus, evaluation of $\mathrm{SiPM}$ for a non-terrestrial cosmic ray detecting telescope, or collection of telescopes, is the goal of the Silicon photomultiplier Elementary Cell Add-on (SiECA) camera. Designed as an ancillary device to be easily attached to existing fluorescence telescopes, utilizing the same optics, internal voltage generation from a 5V supply, internal or external clock and external trigger, serial USB data and communication line, SiECA can be easily mounted and evaluated at any operational cosmic ray telescope provided available space. This capability allowed the SiECA camera to be added to the Extreme Universe Space Observatory (EUSO) Super Pressure Balloon (SPB) mission payload for flight launched in April 2017 [6].

\section{Design \& Construction}

The SiECA camera is constructed of three printed circuit boards: the Elementary Cell (EC) front board supporting the four 64 channel Multi-Pixel Photon Counters (MPPC) sensors (Hamamatsu S13361-3050AS-08), and the two readout boards called the Data Acquisition (DAQ) and Mezzanine (Mezz) boards housing the eight ASIC (Weeroc Citiroc 1A) chips, FPGA (Spartan 6 XC6SLX100-2FGG676I), eight bias voltage generators (Hamamatsu C11204-02), interface control chipsets (USB and LVDS) and other required analog and digital logic components. Each of

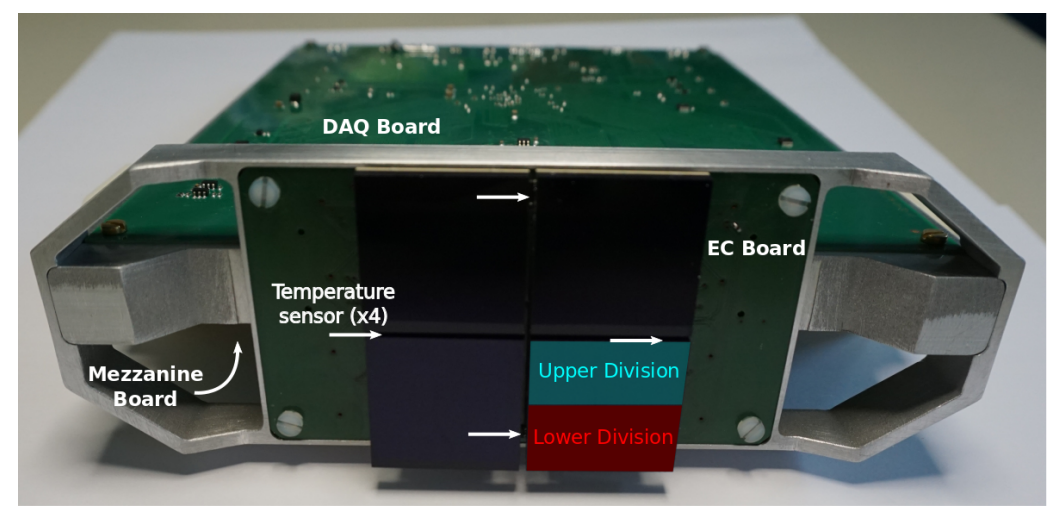

Figure 1: SiECA EC board with BG3 filter covered MPPC sensor arrays. Upper and Lower divisions indicate example bias and ASIC partitioning of each sensor. Aluminum EC-Board frame replaced with black 3D printed plastic for improved analog isolation in flight

the bias supplies and ASIC chips supply and process signal from half of one of the MPPC sensors. This division is such that each MPPC sensor is separated horizontally yielding two sections of 8 channels horizontally and 4 vertically. 


\subsection{Mechanical Frame and Mount}

To ensure rigidity of the circuit board assembly, an aluminum frame was machined. This frame securely houses the DAQ and Mezzanine boards within recessed positions and M2.5 tapped holes allow the boards to be bolted to the frame. The DE9 Power and DE15 LVDS Signal connections are secured in cutouts at the rear of the DAQ/Mezz frame. To improve analog isolation at the EC board, the EC board support frame was replaced with a non-conductive, black 3D printed plastic version. Thickened regions of the DAQ/Mezz frame have two M2.5 mounting holes in each side for attachment of the necessary brace to properly align and secure the camera to the host system. Due to the off center alignment of the DAQ/Mezz boards to the EC center, orientation of the SiECA camera is highly flexible despite the large structure of the readout boards and can be adapted to many existing situations.

\subsection{Sensors and Front End}

The EC board holds the four MPPC arrays and four temperature sensors. Each MPPC is an $8 \times 8$ array of $3 \times 3 \mathrm{~mm}^{2}$ channels. Connection between the sensor and EC board is via a pair of high density SAMTEC connectors which also provide sufficient mechanical rigidity for flight. Testing of SiPM utilizing the Single Photon Calibration stand at KIT (SPOCK) provides full characterization of gain dependence on bias, dark current dependence on bias and photon detection efficiency [7]. Measurements were carried out at room temperature for array characterization over the course of one month with each channel taking approximately 30 minutes to be fully tested [8]. In parallel, testing of the filter gluing process was undertaken. Square prism Schott BG3 filter glass windows

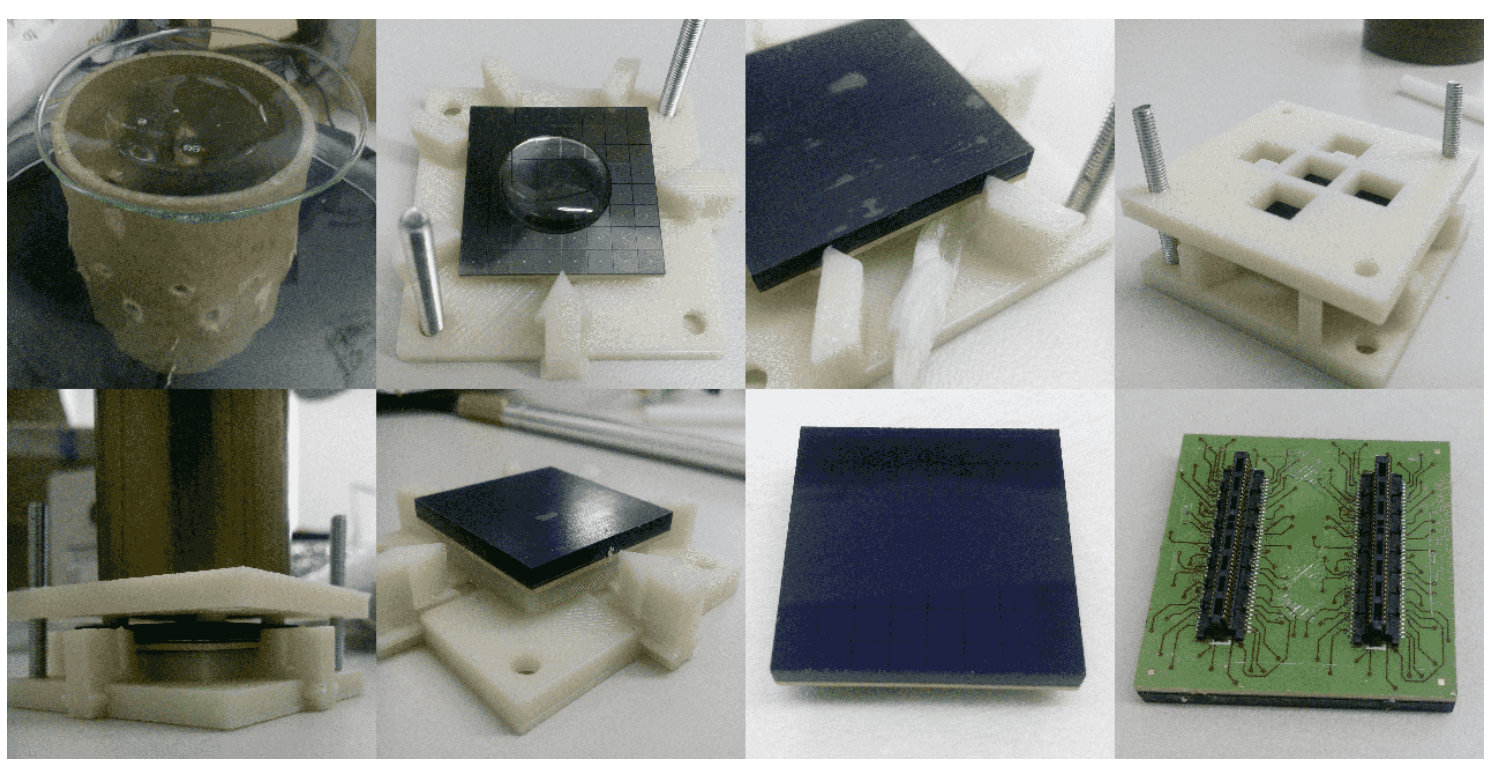

Figure 2: Top left to right then bottom left to right: Degassing mixed EPO-TEK 302-1 (1hr, low vacuum). Transfer degassed epoxy to MPPC in custom alignment frame. Cleaned and taped BG3 filter placed on MPPC and excess epoxy wicked away with filter paper. Compression plate installed. Brass 250 gram mass placed on compression plate for 24 hours. Mass and plate removed with sliding pins pulled away from assembly. Finished MPPC and filter. Underside with some excess epoxy; not a detriment to operation.

$\left(25.8 \times 25.8 \times 2.0 \mathrm{~mm}^{3}\right)$ have been epoxied onto the the silicon MPPC window to selectively allow 
photons in the UV wavelengths of interest to reach the photodiodes while blocking those outside the nitrogen fluorescence spectrum. EPO-TEK 301-2 was used directly on the silicon window and cleaned BG3 filter. This epoxy was selected for its high transmission at short wavelengths $(300-450 \mathrm{~nm})$ [9]. Off-gassing the epoxy is necessary to minimize bubble formation however, small bubbles $\left(\leq 1 \mathrm{~mm}^{2}\right)$ have little effect on photon detection efficiency. Curing the epoxy above room temperature leads to excessive stress and possibly fracture of the filter glass during cooling. No damage has been detected upon cooling the cured sensor and filter assembly to $-40^{\circ} \mathrm{C}$. The four temperature sensors (Texas Instruments LM94021) on the EC board are connected to the bias voltage generators to correctly adjust the provided bias for consistent gain during operation. Supply voltage for the MPPCs, analog signals, and temperature sensor connections between the EC board and the DAQ/Mezz boards are made with Samtec QRF8 \& QRM8 connectors.

\subsection{Data Acquisition and Mezzanine Boards}

The Data Acquisition and Mezzanine boards house all the necessary components for converting the analog MPPC signals into digital signals and then grouping to form event packets for the main operations system to store or transmit. Eight 32-channel SiPM specific Weeroc Citiroc ASICs are required to handle the 256 MPPC channels. The FPGA initializes and operates the system, monitors temperatures and facilitates data transmission. The DAQ and Mezz boards are connected vertically to transmit the digital output of the 4 Mezzanine ASICs to the DAQ mounted FPGA. Eight Hamamatsu C11204-02 bias voltage supplies are used to properly bias the MPPC and adjust for variation in temperatures of the photosensors to stabilize gain throughout operation. Drivers and interfacing components for the USB connection to initialize and receive data from the camera as well as the external clocks and trigger are located along the back edge of the DAQ board.

\section{Data Generation Path}

Ultraviolet photons arriving at the biased MPPC generate a measurable Geiger discharge that passes from the sensor to designated ASIC input channel. The ASIC provides a bias correcting voltage above zero to each channel to flat field the focal surface. The individual channel offset is determined by laboratory tests of each sensor [8]. The signal received at the ASIC is amplified using the high gain amplifier and then discriminated against a threshold corresponding to the first photopeak. Gain and threshold scans were conducted to resolve the optimal settings for low light sensitivity. Both the MPPC calibration and the ASIC settings will be further discussed in the SiECA Calibration section.

When the amplified signal exceeds the discriminator threshold, a digital pulse is generated on the corresponding ASIC output until the analog signal drops back below threshold. Each ASIC has 32 analog inputs and 32 digital outputs. The eight ASICs digital outputs are connected to the FPGA for processing. The FPGA, in flight mode, constantly counts the digital pulses for each channel. This count is written to a 1024 position ring buffer ( 256 Kbytes) at the end of each Gate Time Unit (GTU) $2.5 \mu \mathrm{s}$. When the external trigger is detected, or USB trigger command is received, a user defined number of ring buffer contents at a user defined buffer position (to account for trigger processing delays) are written to the USB FIFO storage for collection by the main system. This packet constitutes one event with a duration equal to the number of GTUs multiplied by the duration 
of each GTU. If an EAS passes through the field of view of the SiECA camera and the latency is properly determined: SiECA will record the event to the system for later analysis. Variation in the GTU length, number of GTUs per event and latency allow for adaptation of SiECA to terrestrial and non-terrestrial telescopes with ease.

The data packet collected with the event contains 38 bytes of system status containing the event count, external trigger count, coarse and fine timestamps from external clock and initialization, read-back of the DAQ control, HV bias control, trigger delay, event length, shutdown register, FPGA firmware revision and operating temperatures of the Citiroc ASICs at time of trigger. These elements all contribute to the understanding of the system at time of measurement so post processing corrections for conditions can be properly assessed and applied.

\subsection{Detector Interface}

Power is provided by a DE9 connection split $+5 \mathrm{~V} / 2 \mathrm{~A}$ on pins $1-5$, ground on pins 6-9. All necessary voltages are generated from this main supply. The DE15 provides LVDS synchronizing 1PPS and $40 \mathrm{MHz}$ clocks as well as the external trigger if utilized. The clock frequencies can be internally generated if necessary and a software trigger is possible over the USB connection. A USB interface with FTDI hardware and firmware is utilized for serial command and data transmission. Changing the GTU length requires changing the $40 \mathrm{MHz}$ clock to the corresponding frequency to ensure proper timing of the FPGA clock synchronization.

\section{SiECA Calibration}

From calibration tests of the MPPC sensors, the channel bias offset in the Citiroc ASIC chips create a detector of uniform gain despite local variation between channels.

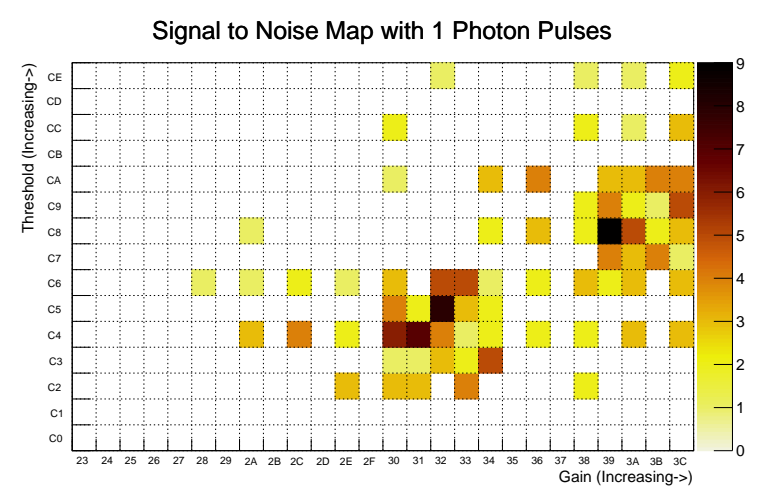

Figure 3: ASIC parameter scan to determine operation gain and threshold setting. Final setting: gain 39, threshold C8. Gain range $[0,3 \mathrm{~F}]$, Threshold range $[0,3 \mathrm{FF}]$

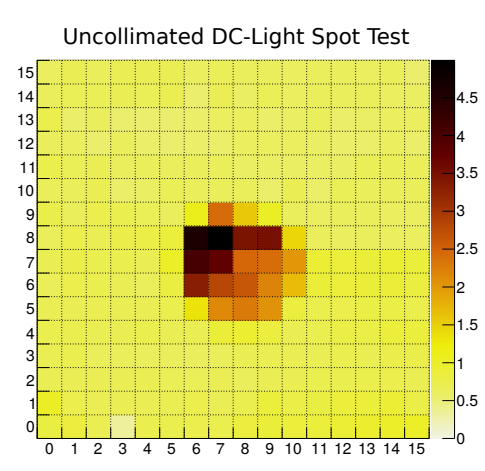

Figure 4: Full camera test with nonuniform light source. Response is average photons detected per GTU

To determine the optimal operational settings, scans over threshold and gain were taken with complete darkness, single photon pulses, and few photon pulses from the calibrated light source in the SPOCK [7]. The light source is pulsed at $1 \mathrm{MHz}$ with the collimator output directed onto a single channel of the SiECA focal surface. A full event (128 GTUs, each $2.5 \mu s$ ) is taken after the reference photodiode measurement has stabilized. Fig. 3 shows the signal to noise ratio for 
each measured gain and threshold setting. In this case, the signal is taken to be the counts on the illuminated channel minus the average counts on a non-illuminated channel. The noise is taken to be the average counts on a non-illuminated channel. As the dark count rate is effectively uniform across the array of channels, this simplified calculation is sufficient to identify the ASIC settings for optimal sensitivity for single photon signals.

Measurement of low intensity DC light source at a distance of $20 \mathrm{~cm}$ from the uncollimated SPOCK aperture shows the extent of optical spreading with the most intense light slightly offcenter from the beam, Fig. 4. This is due to non-exact alignment of the light source and camera center and the $1 \mathrm{~mm}$ spacing between MPPC arrays. Fig. 4 shows the expected uniformity after bias voltage correction for uniform gain outside of the light spot and across the focal surface when not illuminated. The lower response in the upper right area of the light spot is due to a single ASIC threshold offset being improperly set. This issue was fixed before flight.

Measurement at ambient temperatures provide insight into the sensitivity of the camera to single photon emissions. To ensure operation during flight, the SiECA camera was tested for functionality within a temperature chamber and was able to turn on and operate at $-40^{\circ} \mathrm{C}$. Testing of optical response at low temperatures was not possible as the thermal chamber was not integrable in the SPOCK dark chamber [7].

\section{Simulation of SiECA with ESAF}

A preliminary effort to implement SiECA in ESAF to understand the merit of including SiECA with the PDM camera in EUSO-SPB is complete. The simulation of SiECA performance and location in the detector field of view remains very preliminary, however, it is instructive to understand its utility. The SiECA module was added considering it as a new Elementary Cell with a detection efficiency $\sim 30 \%$ higher compared to the standard PDM. Fig. 5 shows an example of a proton event

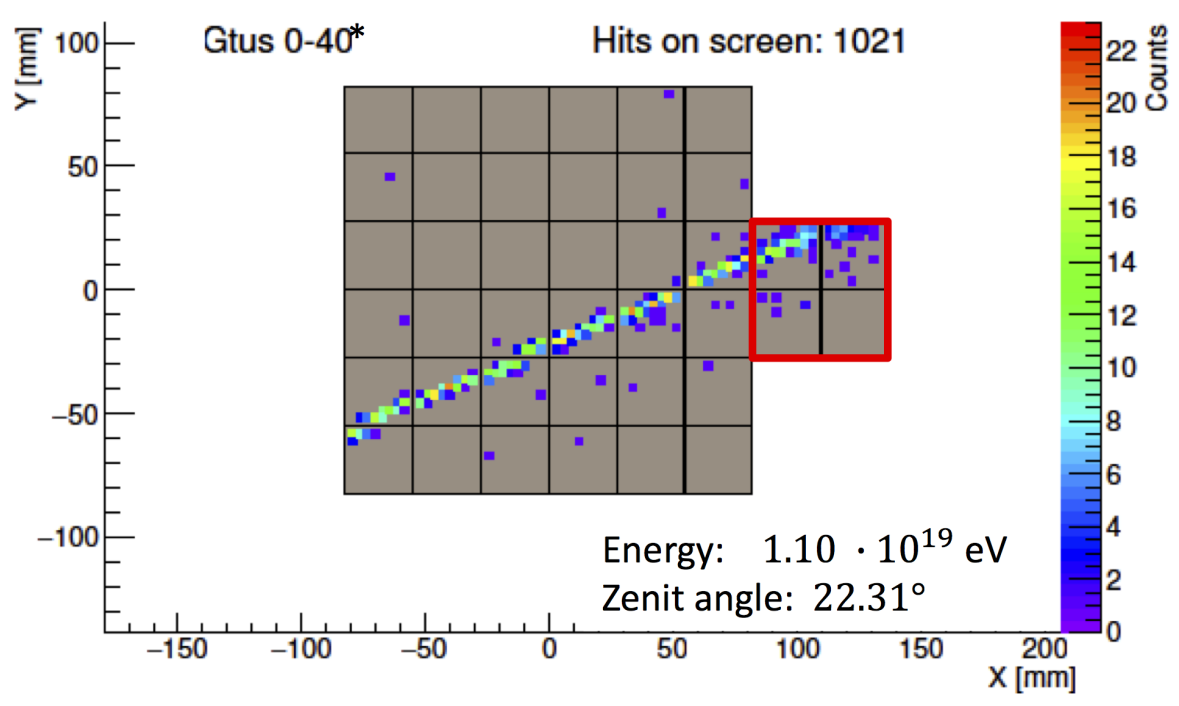

Figure 5: Simulation of a proton event of energy $1.1 \times 10^{19} \mathrm{eV}$ going through the FoV of the detector with a zenith angle $\theta=22.3^{\circ}$. No background is added to the plot. 
of energy $1.1 \times 10^{19} \mathrm{eV}$ going through the FoV of the detector with a zenith angle $\theta=22.3^{\circ}$ which crosses both PDM and SiECA FoV. No background has been added to the plot.

Using the same approach defined in [10], EAS were simulated in clear and cloudy conditions according to the duration and flight conditions experienced in SPB flights 2015 and 2016. Results showed that in $\sim 10 \%$ of the events the EAS signal detected by SiECA was at least $20 \%$ of the total signal detected by the instrument, which indicates that in these events the presence of SiECA would give a significant contribution in the definition of the EAS track.

\section{Flight on EUSO-SPB Mission}

First deployment tests occurred with the launch of the 2017 NASA Super-Pressure Balloon mission carrying the JEM-EUSO prototype telescope, EUSO-SPB [6]. SiECA was attached to the side of the Photo-Detection Module (PDM, EUSO-SPB's main detector) so as to be in the same focal plane and make use of the optics constructed for the mission. As SiECA is designed to evaluate the capability of MPPC for cosmic ray detection, this parallel testing campaign provides direct comparison of MPPC and MAPMT under identical measurement conditions.

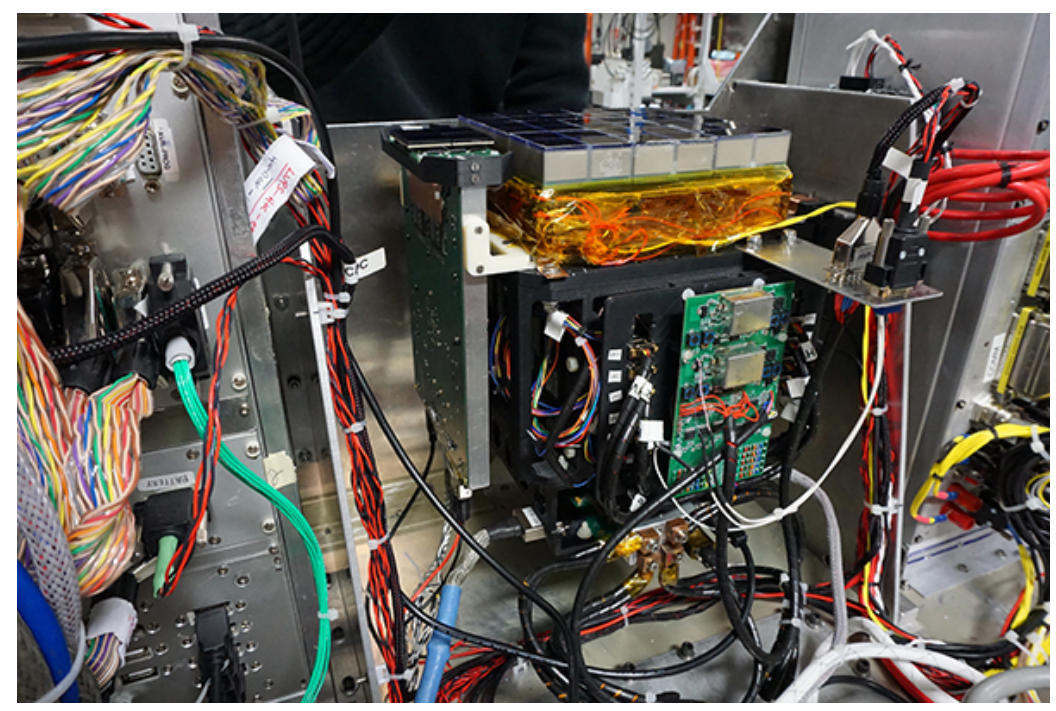

Figure 6: SiECA camera mounted adjacent to the PDM in the Electronics Crate of the EUSO-SPB telescope. Note that the device is upside down relative to operation orientation.

Power is delivered by a commercial DC-DC converter to step down the available battery supply (26-32V) to the required 5V, current limits are imposed at the raw supply side and at the DC-DC to prevent excessive current draw. Connection of the LVDS DE15 is made with the main Clockboard of the EUSO-SPB Data Processor stack [11]. The USB command and data line is connected to the EUSO-SPB Central Processing Unit for initialization and data storage and transmission. Use of 3D printed mounting brace (off-white plastic between SiECA and PDM) provides thermal and electrical isolation. This frame is sufficient to support the camera with minimal torsion and does not interfere with the PDM or PDM electronics.

Preliminary evaluation of the measurements made by SiECA during flight show that all channels of the camera are responding and sensitive to low intensity light. Due to the complications 
of the 2017 NASA Super Pressure Balloon campaign, search for cosmic ray and other interesting signatures in the recorded data is ongoing. Complications during flight, compounded by the unexpected trajectory, concerning electrical interference, seemingly from the SiECA camera, causing instability in the PDM is also to be assessed to determine the cause of this noise and methods for its mediation.

\section{Conclusion}

The development of the SiECA camera and subsequent test flight on the EUSO-SPB mission has provided extensive information about the operation of SiPM at the low temperatures and pressures of the upper atmosphere. Measurements made will be used to characterize both the background UV seen during the flight as well as further improve the camera for future tests. Technical aspects of the camera construction remain to be completely addressed and design of a bi-dimensional expandable unit for larger telescope design is ongoing. Generally, the use of silicon based photomultipliers for downward looking fluorescence and Cherenkov UHECR EAS signatures is promising and the benefits from use of a more robust, compact, low voltage device represent the future of non-terrestrial cosmic ray research.

\section{References}

[1] M. Ricci [JEM-EUSO Collaboration], “The JEM-EUSO Program,” J. Phys. Conf. Ser. 718 (2016) no.5, 052034. doi:10.1088/1742-6596/718/5/052034

[2] A. Aab et al. [Pierre Auger Collaboration], "Muon counting using silicon photomultipliers in the AMIGA detector of the Pierre Auger observatory," JINST 12 (2017) no.03, P03002 doi:10.1088/1748-0221/12/03/P03002 [arXiv:1703.06193 [astro-ph.IM]].

[3] G. Ambrosi et al. [CTA Consortium], "Silicon Photomultipliers and front-end electronics performance for Cherenkov Telescope Array camera development,” Nucl. Instrum. Meth. A 845 (2017) 8. doi:10.1016/j.nima.2016.04.050

[4] T. Niggemann et al., "Status of the Silicon Photomultiplier Telescope FAMOUS for the Fluorescence Detection of UHECRs," arXiv:1502.00792 [astro-ph.IM].

[5] S. Kunwar, [IceCube Collaboration], “The IceTop - Gen2 Scintillator Upgrade”, PoS (ICRC2017)

[6] L. Wiencke et al. [JEM-EUSO Collaboration], "EUSO-SPB Mission and Science in Proc. 35th ICRC," (Busan), (2017).

[7] M. Karus, F. Bisconti, T. Huber, A. Ebersoldt, A. Haungs, H. Schieler and A. Weindl, "Ground Calibration of MAPMT and SiPM for JEM-EUSO,” PoS ICRC 2015 (2016) 612.

[8] M. Renschler, “SiPM + MPPC Calibration”. Thesis. KIT-IKP. 2016.

[9] Epoxy Technology, "EPO-TEK 301-2 Tech Data Sheet,"(April 2015).

[10] F. Fenu et al. (JEM-EUSO Coll.), Proceedings $35^{\text {th }}$ ICRC, Busan, \#... (2017).

[11] G. Osteria et al. [JEM-EUSO Collaboration], "The Data Processor System of EUSO-SPB" Proceedings 35th ICRC, Busan, (2017). 